\title{
Approaches to management of beta-thalassemia intermedia
}

\author{
Joseph E. Maakaron, Ali T. Taher \\ Department of Internal Medicine, Division of Hematology \& Oncology, American University of \\ Beirut Medical Center, Beirut, Lebanon
}

\begin{abstract}
Thalassemia intermedia is a genetically diverse group of diseases that is the result of an imbalance in the production of the alpha and beta chains with ensuing chronic hemolysis, ineffective erythropoiesis, and iron overload.Resulting complications include bone changes, hypercoagulability, and end-organ damage due to iron overload. This decade has witnessed major breakthroughs in the management of thalassemia. In this article, we examine these novelties in therapy including iron chelation therapy, stem cell transplant, and gene therapy.Iron chelation therapy has been revolutionized with the advent of deferasirox, a once-daily oral iron chelator, that has been shown to be safe and efficacious.Gene therapy was also at the core of this revolution with the discovery of novel gene elements and viral vectors allowing for better control and improved outcomes.
\end{abstract}

\section{Pathophysiology}

Beta-thalassemia is an inherited genetic hemoglobin disorder characterized by decreased synthesis of the beta globulin of hemoglobin. The alpha and betachains in a normal red blood cell (RBC) should be in stoichiometric proportion. Underproduction of the beta chains in beta-thalassemia causes excess unstable alpha chains to deposit in the RBC.This increases the oxidative damage to the membrane andcauses subsequent lysis of the cells. The premature death of the RBCs by this process is called ineffective erythropoiesis (1).The bone marrow will react to compensate for the anemia resulting in characteristic deformities of the skull and face because of hypertrophy.Cortical thinning and pathological fractures are also common $(2,3)$. The primary determinant of the anemia is the degree of ineffective erythropoiesis rather than the degree of hemolysis, which only plays a secondary

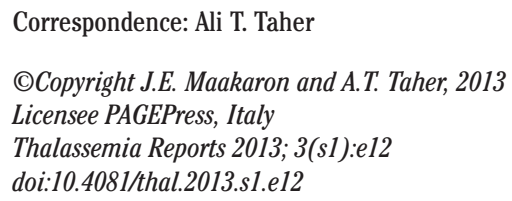

This article is distributed under the terms of the Creative Commons Attribution Noncommercial License (by-nc 3.0) which permits any noncommercial use, distribution, and reproduction in any medium, provided the original author(s) and source are credited.

Parts of this work were presented at the

"3rd Pan-European Conference on Haemoglobinopathies and Rare Anaemias", Limassol (Cyprus), 24-26 October 2012.
role(4).Hemolysis is linked to the hypercoagulable state in TI (5) leading to silent infarcts (6) and pulmonary hypertension. The anemia and the ineffective erythropoiesis will increase intestinal iron absorption leading to iron overload, which causes heart failure, endocrine abnormalities and others. Thus, ineffective erythropoiesis, chronic hemolysis, and iron overload are the factors behind all the complications of TI.

As the medical care we provide for thalassemia patients improves, the life span of the patients is also increasing. This gives us a chance to witness more and more complications. We have shown that TI patients are at a higher risk of thrombosis than TM patients. Out of 2190 TI patients, $3.9 \%$ experienced a thrombotic event compared to only $0.9 \%$ of $6,670 \mathrm{TM}$ patients (7). The events were mainly venous and were divided into deep vein thrombosis (40\%), portal vein thrombosis (19\%), stroke (9\%), pulmonary embolism (12\%), and others (20\%). What's more striking is that splenectomised patients were found to be at a higher risk of thrombosis than non-splenectomised patients. Patients with low hemoglobin concentration $(<9 \mathrm{~g} / \mathrm{dl})$ and non-transfused patients were also found to be at higher risk of thrombosis. Studies are still lacking in this subject but there are several proposed mechanisms. RBC remnants express negatively charged phosphatidylserine residues on their membranes which act as procoagulants, initiating thrombosis $(8,9)$. Activation of platelets, endothelial cells, monocytes, and depletion of antithrombotic factors are also incriminated along with the cardiac, endocrine, and hepatic dysfunction(10). Other risk factors also include age (11), previous thromboembolic events, and family history.It is important to recognize these risks in our patients and treat accordingly since if left untreated, the recurrent thromboembolic phenomena can lead to pulmonary hypertension with ensuing secondary heart failure (12), in addition to silent brain abnormalities $(6,13)$. Recommended therapies include aspirin or other platelet anti-aggregants in the case of thrombocytosis, or anticoagulant agents such as low molecular weight heparin in patients with documented thrombosis or for those undergoing surgery. Blood transfusions might be considered as they dilute the circulating damaged RBCs. More studies are needed to establish a risk-assessment model that would stratify the patients according to their risks and guide a tailored therapy to this issue.

Pietrangelo (14) compared the process of iron regulation to that of glucose regulation. Even though our precise knowledge of the elements involved in iron regulation is still quite lacking, this simile holds in many aspects, with hepcidin being the counterpart of insulin. Iron is controlled by a negative feedback loop. Inflammation and excess plasma iron, just like excess glucose, is a stimulant for the transcription of hepcidin. Hepcidin interacts with ferroportin - the iron exporter - on the basolateral surfaces of hepatocytes and the intestinal epithelium and causes its internalization and degradation (15). This traps iron inside the cells and makes it less available in the blood stream. In the hepatocytes, macrophages, and other cells of the reticuloendothelial system, it is stored in its ferritin-bound form for later use, just like glycogen. In the endothelial cells, intracellular iron is shed with the shedding of the epithelium. Hypoxia, anemia, and a demand for erythropoiesis suppress hepcidin, making more iron avail- 
able for the bone marrow through increased intestinal absorption and better recycling of catabolic iron from the reticuloendothelial system (16). In fact, the ineffective erythropoiesis is an unrelenting signal to downregulate hepcidin by increasing expression of growth differentiation factor 15 (GDF15) and hypoxia-inducible transcription factors (HIFs) (16), thus dumping more iron in the plasma through increasing intestinal absorption and depleting the macrophages of their iron stores. After all the iron saturates the transferrin stores, it is transported as the toxic non-transferrin-bound-iron. The end result is deposition in the parenchyma of the liver, heart, endocrine organs, and others. Coming back to the analogy, thalassemia intermedia is a lot like diabetes mellitus type 2. Hormonal failure, manifesting either as decreased secretion or as decreased sensitivity, is the culprit in both.

Body iron levels are usually assessed by ferritin values. However, studies have shown that ferritin underestimates the total iron burden in TI, or at least does not increase as much as TM (17). For the same value of LIC, ferritin values in TI patients were significantly lower than TM patients. A proposed mechanism for this is that iron in transfused patients is preferentially distributed to the reticuloendothelial system, thus ferritin is more readily synthesized and exported(16). This is in contrast to transfusion-independent TI patients, where the low hepcidin depletes the reticuloendothelial system of its iron stores, thus ferritin will be low.Other methods of evaluating body iron stores include determination of liver iron concentration by biopsy or more recently by non-invasive techniques such as R2 MRI.

The main indications for splenectomy in TI are poor growth and development, increased transfusion demand, hypersplenism, and splenomegaly (18). However, splenectomy appears to contribute to an increased risk of thrombosis $(9,19)$. Splenectomy is now less common than before and is performed later in life (20). Gallstones are common in TI because of the ineffective erythropoiesis and peripheral hemolysis. That is why the gallbladder should be inspected during splenectomy to avoid further complications.

Extramedullary hematopoiesis (EMH) is when erythropoietic tissue outside the confines of marrow spaces in bones increase to compensate for the chronic state of anemia that accompanies TI. This will lead to the formation of erythropoietic masses that will primarily affect the spleen, liver, and lymph nodes (20). These masses can cause local symptoms such as neurological symptoms and spinal cord compressions $(21,22)$. There is even one case report on hematopoietic masses causing recurrent pleural effusion(23). Splenic enlargement can cause symptoms such as early satiety, left upper quadrant pain, and even raises concerns about splenic rupture(18). Extramedullary hematopoiesis is routinely managed by radiotherapy, hydroxyurea, or transfusion therapy $(21,24,25)$.

Pulmonary hypertension (PH) is defined as pulmonary artery pressure of $>25 \mathrm{~mm} \mathrm{Hg}$ at rest or $>30 \mathrm{~mm} \mathrm{Hg}$ during exercise(26). $\mathrm{PH}$ is quite prevalent in hemolytic anemias in general and in TI, the prevalence is estimated to be around 59\%.This, in turn, contributes to an increased incidence of congestive heart failure(27).The etiology of PH in TI is multifactorial, and its pathogenesis is complicated, but as we gain more insight into this disease, we are better able to treat it and even prevent it. PH results from interplay of vasoconstriction, vascular smooth muscle proliferation, and dysfunctional endothelium with resulting thrombosis, all of which contribute to luminal narrowing and right-sided failure. Major risk factors include advancing age and a history of splenectomy (28). Autopsy studies in thalassemic patients have revealed common histopathological findings that include plexiform and concentric medial hyperplastic pulmonary vascular lesions, and in situpulmonary artery thrombosis $(29,30)$. The factors that culminate in these pathologies are most likely the result of the long-term sequelae of splenectomy, red cell membrane pathology, coagulation abnormalities, excess arginase activity, low nitric oxide bioavailability, platelet activation, oxidative stress, iron overload, and chronic hemolysis (31-
35). The correlation of ferritin with $\mathrm{PH}$ is still controversial with one study indicating no correlation (36) with another study reporting a strong correlation between an elevated tricuspid regurgitant jet velocity (TRV) and serum ferritin(37).Hemolysis disables the arginine-nitric oxide pathway by releasing erythrocyte arginase(38) and cell-free hemoglobin(33). With the consumption of nitric oxide and arginine, endothelial dysfunction ensues, and along with the intravascular hemolysis, this will create a procoagulant state (39). Iron overload, and the subsequent oxidative stress, can also contribute to increasing the pulmonary vasculature resistance through various mechanisms. They induce pulmonary fibrosis and affect the pump itself through cardiac siderosis(40). The hypoxemia, along with the chronic anemia, will also exacerbate the vasoconstriction(28).

Currently, there are no guidelines for the treatment of PH in TI specifically. In one small trial, $\mathrm{PH}$ was absent in patients taking hydroxyurea suggesting a protective role, but further studies are needed (41). Two small studies reported improvement in exercise tolerance in response to sildenafil $(42,43)$. General recommendations for the management of PH now are adequate treatment of thalassemia, any precipitating factors or associated diseases, in addition to supportive measures(28). A multidisciplinary approach is needed and a cardiopulmonary specialist should be on board.

TI patients are also at risk for other complications. Leg ulcers occur more commonly in older patients. Leg ulcers develop in some patients with low hemoglobin and do not develop in others, even though they are maintained at the same amount of fetal hemoglobin, implying that it is definitely an interplay of several factors. The skin might be at a lower oxygen tension due to the anemia, making it fragile and more prone to break upon minimal trauma. The healing process would also be impaired under these conditions. These are usually managed by an intense transfusion regimen to elevate the hemoglobin concentration by a minimum of $2 \mathrm{~g} / \mathrm{dl}$. Other possible interventions include elevating the end of the bed so as to keep the lower limbs at a level above the heart. This provides better venous return and increases the perfusion pressure of the affected area(20). Zinc supplementation (44), pentoxifylline, and hydroxyurea, without or without erythropoietin (45) are also possible options.

While endocrine dysfunction can be quite common in TM, it is quite rare in TI. Patients generally experience late puberty, but their sexual development is normal and their fertility preserved. However, hypothyroidism can be a late occurrence sometimes(20).

Viral hepatitis is much rarer in TI than in TM due to the fact that TI patients are less often transfused. Nevertheless, the risk is not absent and careful monitoring should be undertaken. With the advent of better medical care and chelation therapy, TI patients are surviving longer and new complications such as hepatocellular carcinoma are emerging (Figure 1, Table 1)(46).

\section{Management}

The management of TI has truly come a long way this decade especially the breakthroughs in oral iron chelation therapy, gene therapy, and the promised breakthroughs in JAK2 inhibitors. Transfusion is definitely the only available salvage therapy when severe symptoms of anemia set in, including developmental delay in the pediatric age group. Associated risks include alloimmunisation, which is relatively common in TI, especially if transfusions are instituted after the age of 12 months (47). Rhesus and Kellphenotyping is recommended prior to transfusion (48), with some physicians advocating a short course of steroids for 3 - 5 days concomitantly, even though this remains controversial.

Iron chelation therapy is the mainstay of treatment of TI and studies 


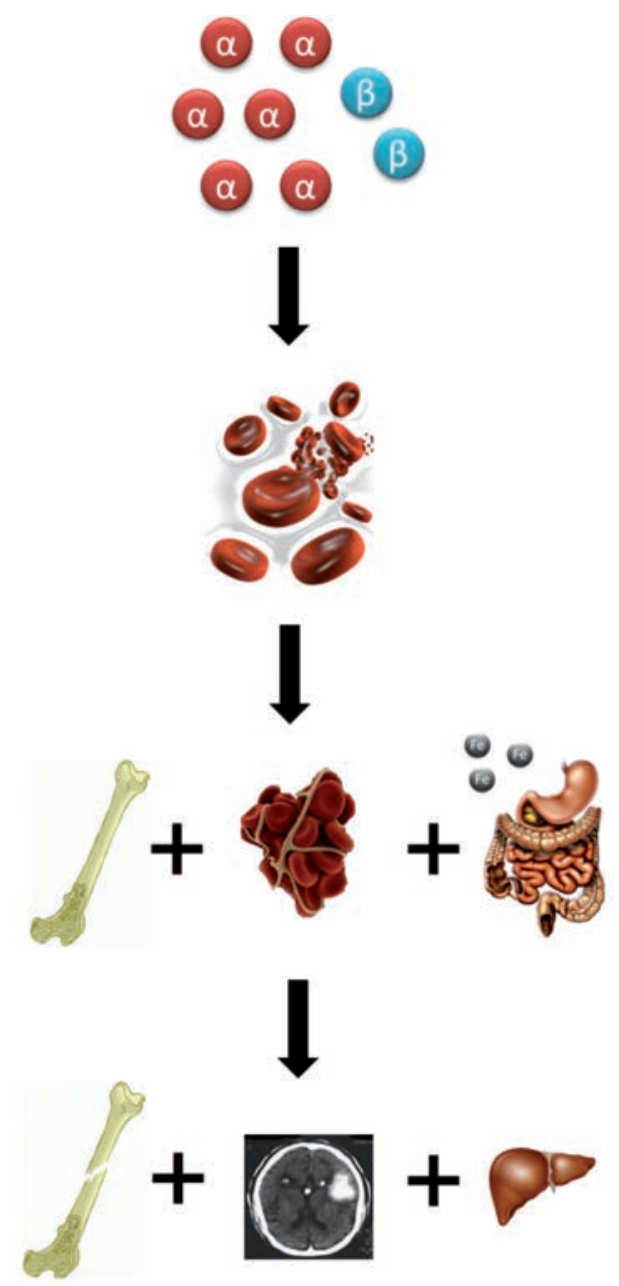

Figure 1. Pathophysiology of thalassemia intermedia in a nutshell. An imbalance in the ration of alpha to non-alpha (beta) chains will lead to cell lysis due to oxidative stress with ensuing chronic hemolysis and ineffective erythropoiesis. This leads to bony changes, hypercoagulability, and increased gastrointestinal iron absorption. The end result being osteoporosis and fractures, silent brain infarcts and pulmonary hypertension because of the clots formed, and iron overload manifesting in increased liver iron concentration. demonstrating the efficacy and benefits of this study are increasing by the day. The rate of iron loading in TI is variable and changes with transfusional load. Iron loading in non-transfused patients is estimated to be 2 - 5 grams per year (49) compared to 7.5 - 15.1 grams per year for transfused patients (50).Iron loading should always be assessed by liver iron concentration (LIC), preferably by non-invasive MRI imaging, and iron chelation therapy initiated accordingly. Previously, an LIC of 7 $\mathrm{mg} \mathrm{Fe} / \mathrm{g}$ dry weight was used to initiate therapy; however, a recent study has found that an LIC of $7 \mathrm{mg} \mathrm{Fe} / \mathrm{g}$ dry weight is associated with vascular complications and an LIC of $6 \mathrm{mg} \mathrm{Fe} / \mathrm{g}$ dry weight is associated with endocrine comorbidities, meaning that complications would have already set in, and chelation should start before that (51).

The gold standard remains desferrioxamine injections which is given subcutaneously or intravenously for prolonged periods of time and is associated with significant patient discomfort, decreased quality of life, and non-compliance $(52,53)$ despite significant mortality and morbidity reductions (54). Deferasirox, a once-daily oral iron chelator, has been found to be safe and efficacious over a wide range of transfusion-dependent anemias and heavily iron-loaded patients $(55,56)$. A pilot study has also shown it to be effective in reducing iron burden in TI patients (57). A large randomized, double-blind, placebo-controlled study is ongoing to determine the efficacy and safety of deferasirox in TI and other non-transfusion-dependent anemias.

A novel oral iron chelator is also under development for clinical use. The molecule is still in phase I clinical trials, but the results seems promising(58). The authors state that extensive preclinical Extensive preclinical toxicological studies demonstrated a higher no-observableadverse-effect level (NOAEL) compared to deferasirox.

Hematopoietic stem-cell transplantation is essentially curative in properly selected candidates. Good candidates with high success rates include pediatric patients who have not developed complications such as viral hepatitis or severe iron overload and who receive HLA-identical related donor stem-cell transplants. The event-free survival for beta-thalassemia patients reported from several groups is $80-90 \%$ with less than $10 \%$ mortality and minimal morbidity, apart from impaired fertility (59-62).The problem is in the availability of donors. Disease-free survival is lower, and morbidity and mortality is higher with unrelated haploidentical donors (63-65). Definitive hematopoietic stem cells can be obtained from cord blood. Several studies have assessed the use of cord-blood transplantation for patients with thalassemia (61), and the outcomes of related cord-blood stem cell transplantation for beta-thalassemia are approaching those of conventional bone marrow transplantation with disease-free survival of

Table 1. Complications of thalassemia intermedia.

Hypercoagulability caused by circulating procoagulant molecules and exacerbated by splenectomy

Iron overload caused by increased GI absorption

Splenomegaly and hypersplenism as a result of ineffective erythropoiesis

Cholelithiasis due to chronic hemolysis

Extramedullary hematopoiesis due to anemia and ineffective erythropoiesis

Pulmonary hypertension probably due to microthrombi in the pulmonary vasculature

Leg ulcers

Endocrine dysfunction due to iron overload

Hepatitis due to transfusion therapy
Acetyl salicylic acid or other anti-platelet aggregant for thrombocytosis

LMWH for previous thrombosis

Iron chelation therapy and regular assessment of iron loading

Splenectomy - with careful consideration of the increased adverse events Cholecystectomy

Radiotherapy, hydroxyurea, or transfusion therapy

Hydroxyurea

Sildenafil still under study

Hypertransfusion and elevation of the LL

Zinc supplementation, pentoxifylline, and

hydroxyurea \pm erythropoietin

Iron chelation therapy and regular follow-up

Using effective transfusion regimens

Careful monitoring for hepatocellular carcinoma 
90\%. A cord-blood bank will also allow unrelated transplants to take place, but data is still scarce (66-68). A recent review cited a personal communication from a member of the Eurocord cooperative group that this is feasible, albeit less successful than HLA-compatible cord-blood transplant from a sibling (69). The physician, patient, and everybody concerned should be involved in the transplantation decision given the associated morbidity, mortality, and cost of the procedure.

Thalassemia is an ideal disease for gene therapy since its genetic defect only affects the erythroid lineage in the hematopoietic system. The concept it is simple, albeit the mechanism is technically demanding. Hematopoietic stem cells are harvested from the patient and infected with a virus carrying the beta-globin gene along with its upstream regulatory elements, and then infused back after myelosuppression. The fact that the patient's own cells are used overcomes the problem of scarcity of donors and associated immunological complications.

This field has also witnessed a great deal of progress lately and many obstacles have been reduced. A lot of work has been put to develop viral constructs that are able to accommodate all the sequences needed for the expression of the beta-globin gene. The position where the construct integrates also has grave effects. Therefore, boundary elements are now frequently included in the genetic complexes. Boundary elements are sequences that flank the gene and delimit the activity of the regulatory elements of that gene (70). Another setback was the associated risk of leukemia with the use of retroviral vectors. Therefore, lentiviral vectors were developed and they have proven successful in mouse models both in TM and TI (71-76). A clinical trial to test this lentiviral vector has already begun in 2007 on two patients $(77,78)$. One of the patients needed rescue with non-manipulated cells while the other achieved $10 \%$ engraftment and was able to become transfusion independent. The future of gene therapy probably lies in induced pluripotent stem cells, which are human somatic cells that are reprogrammed to form multipotent stem cells $(79,80)$.

Ineffective erythropoiesis is when erythroid progenitor cells fail to mature, die in the process, or develop into abnormal erythrocytes that eventually die prematurely. This process is due to chain imbalances in the erythroid precursors and is the root of the pathophysiology in thalassemia. The premature erythrocytes produced undergo premature apoptosis, leading to intravascular and medullary hemolysis. Ineffective erythropoiesis also causes a compensatory expansion of the erythron, even though it still fails to maintain a normal numbers of erythrocytes. This will produce characteristic deformities of the skull and spine, osteoporosis, and bone demineralization. The spleen will also enlarge, thus exacerbating the anemia by sequestration of red blood cells. Ineffective erythropoiesis will also downregulate hepcidin, thus instituting a state of iron overload. Data showed that in ineffective erythropoiesis, there is an accumulation of proliferating erythroid progenitors, and there was an increase in the percentage of erythroid cells in the S-phase. The thalassemic cells were associated with the expression of certain cell-cycle regulatory genes such as EpoR, Jak2, cyclin-A, Cdk2, Ki-67 and the antiapoptotic protein Bcl-XL, and differentiated less. In thalassemic mice, a Jak2 inhibitor reversed ineffective erythropoiesis and decreased spleen size with limited effect on anemia. Jak2 inhibitors would be expected to limit or reduce splenomegaly, thus improving anemia and delaying the need for splenectomy - a procedure which carries its own complications. A decrease in ineffective erythropoiesis is also expected to upregulate hepcidin, thus improving the metabolism of total body iron content and decreasing endorgan damage(81-83).

\section{References}

1. Olivieri, N.F., The beta-thalassemias. N Engl J Med, 1999. 341(2): p. 99-109.
2. Camaschella, C. and M.D. Cappellini, Thalassemia intermedia. Haematologica, 1995. 80(1): p. 58-68.

3. Cappellini, M.D., et al., Thalassemia intermedia: clinical aspects and management. Haematologica, 2001. 86: p. 194-196.

4. Taher, A., H. Isma'eel, and M.D. Cappellini, Thalassemia intermedia: revisited. Blood Cells Mol Dis, 2006. 37(1): p. 12-20.

5. Ataga, K.I., M.D. Cappellini, and E.A. Rachmilewitz, Beta-thalassaemia and sickle cell anaemia as paradigms of hypercoagulability. Br J Haematol, 2007. 139(1): p. 3-13.

6. Musallam, K.M., et al., Brain positron emission tomography in splenectomized adults with beta-thalassemia intermedia: uncovering yet another covert abnormality. Ann Hematol, 2011.

7. Taher, A., et al., Prevalence of thromboembolic events among 8,860 patients with thalassaemia major and intermedia in the Mediterranean area and Iran. Thromb Haemost, 2006. 96(4): p. 488-91.

8. Eldor, A. and E.A. Rachmilewitz, The hypercoagulable state in thalassemia. Blood, 2002. 99(1): p. 36-43.

9. Cappellini, M.D., et al., Venous thromboembolism and hypercoagulability in splenectomized patients with thalassaemia intermedia. Br J Haematol, 2000. 111(2): p. 467-73.

10. Taher, A.T., et al., Thalassemia and hypercoagulability. Blood Rev, 2008. 22(5): p. 283-92.

11. Taher, A.T., et al., Age-related complications in treatment-naive patients with thalassaemia intermedia. Br J Haematol, 2010. 150(4): p. 486-9.

12. El-Beshlawy, A., et al., Pulmonary hypertension in beta-thalassemia major and the role of L-carnitine therapy. Pediatr Hematol Oncol, 2008. 25(8): p. 734-43.

13. Taher, A.T., et al., Covert brain ischaemia in splenectomised adults with thalassemia intermedia: An emerging entity. Thromb Haemost, 2010. 104(3): p. 652-3.

14. Pietrangelo, A., Hemochromatosis: an endocrine liver disease. Hepatology, 2007. 46(4): p. 1291-301.

15. Nemeth, E., et al., Hepcidin regulates cellular iron efflux by binding to ferroportin and inducing its internalization. Science, 2004. 306(5704): p. 2090-3.

16. Taher, A., C. Hershko, and M.D. Cappellini, Iron overload in thalassaemia intermedia: reassessment of iron chelation strategies. $\mathrm{Br} \mathrm{J}$ Haematol, 2009. 147(5): p. 634-40.

17. Pakbaz, Z., et al., Serum ferritin underestimates liver iron concentration in transfusion independent thalassemia patients as compared to regularly transfused thalassemia and sickle cell patients. Pediatr Blood Cancer, 2007. 49(3): p. 329-32.

18. Taher, A.T., et al., Optimal management of beta thalassaemia intermedia. Br J Haematol, 2011. 152(5): p. 512-23.

19. Cappellini, M.D., et al., Redefining thalassemia as a hypercoagulable state. Ann N Y Acad Sci, 2010. 1202: p. 231-6.

20. Cappellini, M.D., et al., Disorders of Erythropoiesis, Erythrocytes and Iron Metabolism, ed. Carole Beaumont, et al.2009, Genoa, Italy: Forum Service Editore.

21. Chehal, A., et al., Hypertransfusion: a successful method of treatment in thalassemia intermedia patients with spinal cord compression secondary to extramedullary hematopoiesis. Spine (Phila Pa 1976), 2003. 28(13): p. E245-9.

22. Castelli, R., et al., Intrathoracic masses due to extramedullary hematopoiesis. Am J Med Sci, 2004. 328(5): p. 299-303.

23. Taher, A., et al., Extramedullary hematopoiesis in a patient with beta-thalassemia intermedia manifesting as symptomatic pleural effusion. Hemoglobin, 2001. 25(4): p. 363-8.

24. Saxon, B.R., D. Rees, and N.F. Olivieri, Regression of extramedullary haemopoiesis and augmentation of fetal haemoglobin concentration during hydroxyurea therapy in beta thalassaemia. Br J Haematol, 1998. 101(3): p. 416-9.

25. Cario, H., et al., Treatment with hydroxyurea in thalassemia inter- 
media with paravertebral pseudotumors of extramedullary hematopoiesis. Ann Hematol, 2002. 81(8): p. 478-82.

26. Gladwin, M.T., et al., Pulmonary hypertension as a risk factor for death in patients with sickle cell disease. N Engl J Med, 2004. 350(9): p. 886-95.

27. Isma'eel, H., et al., Relation between iron-overload indices, cardiac echo-Doppler, and biochemical markers in thalassemia intermedia. Am J Cardiol, 2008. 102(3): p. 363-7.

28. Morris, C.R. and E.P. Vichinsky, Pulmonary hypertension in thalassemia. Ann N Y Acad Sci, 2010. 1202: p. 205-13.

29. Sonakul, D., P. Pacharee, and K. Thakerngpol, Pathologic findings in 76 autopsy cases of thalassemia. Birth Defects Orig Artic Ser, 1988. 23(5B): p. 157-76.

30. Morris, C.R., M.T. Gladwin, and G.J. Kato, Nitric oxide and arginine dysregulation: a novel pathway to pulmonary hypertension in hemolytic disorders. Curr Mol Med, 2008. 8(7): p. 620-32.

31. Aessopos, A., et al., Thalassemia heart disease: a comparative evaluation of thalassemia major and thalassemia intermedia. Chest, 2005. 127(5): p. 1523-30.

32. Hahalis, G., et al., Heart failure in beta-thalassemia syndromes: a decade of progress. Am J Med, 2005. 118(9): p. 957-67.

33. Morris, C.R., Mechanisms of vasculopathy in sickle cell disease and thalassemia. Hematology Am Soc Hematol Educ Program, 2008: p. 177-85.

34. Singer, S.T., et al., Pulmonary hypertension in thalassemia: association with platelet activation and hypercoagulable state. Am J Hematol, 2006. 81(9): p. 670-5.

35. Morris, C.R., Pulmonary hypertension in thalassemia assessed by echocardiography: a report from base-line data of the thalassemia clinical research network Longitudinal Cohort Study. Blood, 2009. 114: p. 796:(abst 2016).

36. Hagar, R.W., C.R. Morris, and E.P. Vichinsky, Pulmonary hypertension in thalassaemia major patients with normal left ventricular systolic function. Br J Haematol, 2006. 133(4): p. 433-5.

37. Mokhtar, G.M., et al., N-terminal natriuretic peptide and ventilation-perfusion lung scan in sickle cell disease and thalassemia patients with pulmonary hypertension. Hemoglobin, 2010. 34(1): p. 78-94.

38. Morris, C.R., Role of arginase in sickle cell lung disease and hemolytic anemias. Open Nitric 0xide J, 2010. 2: p. 41-54.

39. Setty, B.N., et al., Heme induces endothelial tissue factor expression: potential role in hemostatic activation in patients with hemolytic anemia. J Thromb Haemost, 2008. 6(12): p. 2202-9.

40. Zakynthinos, E., et al., Pulmonary hypertension, interstitial lung fibrosis, and lung iron deposition in thalassaemia major. Thorax, 2001. 56(9): p. 737-9.

41. Karimi, M., et al., Echocardiographic finding in beta-thalassemia intermedia and major: absence of pulmonary hypertension following hydroxyurea treatment in beta-thalassemia intermedia. Eur $\mathrm{J}$ Haematol, 2009. 82(3): p. 213-8.

42. Derchi, G., et al., Efficacy and safety of sildenafil in the treatment of severe pulmonary hypertension in patients with hemoglobinopathies. Haematologica, 2005. 90(4): p. 452-8.

43. Littera, R., et al., Long-term treatment with sildenafil in a thalassemic patient with pulmonary hypertension. Blood, 2002. 100(4): p. 1516-7.

44. Gupta, V.L. and B.S. Choubey, RBC survival, zinc deficiency, and efficacy of zinc therapy in sickle cell disease. Birth Defects Orig Artic Ser, 1987. 23(5A): p. 477-83.

45. al-Momen, A.K., Recombinant human erythropoietin induced rapid healing of a chronic leg ulcer in a patient with sickle cell disease. Acta Haematol, 1991. 86(1): p. 46-8.

46. Mancuso, A., Hepatocellular carcinoma in thalassemia: A critical review. World J Hepatol, 2010. 2(5): p. 171-4.
47. Spanos, T., et al., Red cell alloantibodies in patients with thalassemia. Vox Sang, 1990. 58(1): p. 50-5.

48. Hmida, S., et al., Red cell alloantibodies in patients with haemoglobinopathies. Nouv Rev Fr Hematol, 1994. 36(5): p. 363-6.

49. Pippard, M.J., S.T. Callender, and C.A. Finch, Ferrioxamine excretion in iron-loaded man. Blood, 1982. 60(2): p. 288-94.

50. Federation, T.I. Guidelines for the clinical management of thalassemia. 2004; Available from: http://www.thalassaemia.org/cy/ Publications/htm.

51. Musallam, K.M., et al., Elevated liver iron concentration is a marker of increased morbidity in patients with \{beta\} thalassemia intermedia. Haematologica, 2011.

52. Treadwell, M.J. and L. Weissman, Improving adherence with deferoxamine regimens for patients receiving chronic transfusion therapy. Semin Hematol, 2001. 38(1 Suppl 1): p. 77-84.

53. Cappellini, M.D., Overcoming the challenge of patient compliance with iron chelation therapy. Semin Hematol, 2005. 42(2 Suppl 1): p. S19-21.

54. Cossu, P., et al., Iron overload and desferrioxamine chelation therapy in beta-thalassemia intermedia. Eur J Pediatr, 1981. 137(3): p. 267-71.

55. Cappellini, M.D., et al., Tailoring iron chelation by iron intake and serum ferritin: the prospective EPIC study of deferasirox in 1744 patients with transfusion-dependent anemias. Haematologica, 2010. 95(4): p. 557-66.

56. Taher, A., et al., Efficacy and safety of deferasirox, an oral iron chelator, in heavily iron-overloaded patients with beta-thalassaemia: the ESCALATOR study. Eur J Haematol, 2009. 82(6): p. 458-65.

57. Voskaridou, E., et al., Treatment with deferasirox (Exjade) effectively decreases iron burden in patients with thalassaemia intermedia: results of a pilot study. Br J Haematol, 2010. 148(2): p. 332-4.

58. Rienhoff, H.Y., Jr., et al., A phase 1 dose-escalation study: safety, tolerability, and pharmacokinetics of FBS0701, a novel oral iron chelator for the treatment of transfusional iron overload. Haematologica, 2011. 96(4): p. 521-5.

59. Krishnamurti, L., et al., Hematopoietic cell transplantation for hemoglobinopathies. Curr Probl Pediatr Adolesc Health Care, 2008. 38(1): p. 6-18.

60. Lucarelli, G., et al., Bone marrow transplantation in patients with thalassemia. N Engl J Med, 1990. 322(7): p. 417-21.

61. Pinto, F.0. and I. Roberts, Cord blood stem cell transplantation for haemoglobinopathies. Br J Haematol, 2008. 141(3): p. 309-24.

62. Smiers, F.J., L. Krishnamurti, and G. Lucarelli, Hematopoietic stem cell transplantation for hemoglobinopathies: current practice and emerging trends. Pediatr Clin North Am, 2010. 57(1): p. 181-205.

63. Dini, G., et al., Unrelated donor marrow transplantation: initial experience of the Italian bone marrow transplant group (GITMO). Bone Marrow Transplant, 1996. 17(1): p. 55-62.

64. La Nasa, G., et al., Unrelated donor bone marrow transplantation for thalassemia: the effect of extended haplotypes. Blood, 2002. 99(12): p. 4350-6.

65. Yannaki, E., D.W. Emery, and G. Stamatoyannopoulos, Gene therapy for beta-thalassaemia: the continuing challenge. Expert Rev Mol Med, 2010. 12: p. e31.

66. Boncimino, A., A. Bertaina, and F. Locatelli, Cord blood transplantation in patients with hemoglobinopathies. Transfus Apher Sci, 2010. 42(3): p. 277-81.

67. Hall, J.G., et al., Unrelated umbilical cord blood transplantation for an infant with beta-thalassemia major. J Pediatr Hematol Oncol, 2004. 26(6): p. 382-5.

68. Jaing, T.H., et al., Rapid and complete donor chimerism after unrelated mismatched cord blood transplantation in 5 children with beta-thalassemia major. Biol Blood Marrow Transplant, 2005. 11(5): p. 349-53. 
69. Higgs, D.R., J.D. Engel, and G. Stamatoyannopoulos, Thalassaemia. Lancet, 2011.

70. Gaszner, M. and G. Felsenfeld, Insulators: exploiting transcriptional and epigenetic mechanisms. Nat Rev Genet, 2006. 7(9): p. 703-13.

71. May, C., et al., Successful treatment of murine beta-thalassemia intermedia by transfer of the human beta-globin gene. Blood, 2002 . 99(6): p. 1902-8.

72. Hacein-Bey-Abina, S., et al., LM02-associated clonal T cell proliferation in two patients after gene therapy for SCID-X1. Science, 2003. 302(5644): p. 415-9.

73. Hacein-Bey-Abina, S., et al., Efficacy of gene therapy for X-linked severe combined immunodeficiency. N Engl J Med, 2010. 363(4): p. 355-64.

74. Imren, S., et al., Permanent and panerythroid correction of murine beta thalassemia by multiple lentiviral integration in hematopoietic stem cells. Proc Natl Acad Sci U S A, 2002. 99(22): p. 14380-5.

75. May, C., et al., Therapeutic haemoglobin synthesis in beta-thalassaemic mice expressing lentivirus-encoded human beta-globin. Nature, 2000. 406(6791): p. 82-6.

76. Persons, D.A., et al., The degree of phenotypic correction of murine beta -thalassemia intermedia following lentiviral-mediated transfer of a human gamma-globin gene is influenced by chromosomal position effects and vector copy number. Blood, 2003. 101(6): p. 2175-83.

77. Cavazzana-Calvo, M., et al., Transfusion independence and HMGA2 activation after gene therapy of human beta-thalassaemia. Nature, 2010. 467(7313): p. 318-22.

78. Kaiser, J., Gene therapy. Beta-thalassemia treatment succeeds, with a caveat. Science, 2009. 326(5959): p. 1468-9.

79. Takahashi, K., et al., Induction of pluripotent stem cells from adult human fibroblasts by defined factors. Cell, 2007. 131(5): p. 861-72.

80. Takahashi, K., et al., Induction of pluripotent stem cells from fibroblast cultures. Nat Protoc, 2007. 2(12): p. 3081-9.

81. Taher, A., et al., Stroke in thalassemia: a dilemma. Am J Hematol, 2008. 83(4): p. 343.

82. Rivella, S., Ineffective erythropoiesis and thalassemias. Curr Opin Hematol, 2009. 16(3): p. 187-94.

83. Libani, I.V., et al., Decreased differentiation of erythroid cells exacerbates ineffective erythropoiesis in beta-thalassemia. Blood, 2008. 112(3): p. 875-85.

\section{Oral presentation}

Oral presentation is available online 